\title{
MOD $p$ VANISHING THEOREM OF SEIBERG-WITTEN INVARIANTS FOR 4-MANIFOLDS WITH $\mathbb{Z}_{p}$-ACTIONS*
}

\author{
NOBUHIRO NAKAMURA ${ }^{\dagger}$
}

\begin{abstract}
We give an alternative proof of the $\bmod p$ vanishing theorem by F. Fang of SeibergWitten invariants under a cyclic group action of prime order, and generalize it to the case when $b_{1} \geq 1$. Although we also use the finite dimensional approximation of the monopole map as well as Fang, our method is rather geometric. Furthermore, non-trivial examples of mod $p$ vanishing are given.
\end{abstract}

Key words. 4-manifolds, Seiberg-Witten invariants, group actions.

AMS subject classifications. Primary: 57R57, 57S17. Secondary: 57M60.

1. Introduction. In this paper, we investigate Seiberg-Witten invariants under a cyclic group action of prime order. The Seiberg-Witten gauge theory with group actions has been studied by many authors $[21,7,9,10,16,15,8,5,18]$ etc. Among these, we pay attention to a work by F. Fang [10].

In the paper [10], Fang proves that the Seiberg-Witten invariant of a smooth 4 -manifold $X$ of $b_{1}=0$ and $b_{+} \geq 2$ under an action of cyclic group $\mathbb{Z}_{p}$ of prime order $p$, vanishes modulo $p$ if some inequality about the $\mathbb{Z}_{p}$-index of Dirac operator and $b_{+}$is satisfied, where $b_{i}$ is the $i$-th Betti number of $X$ and $b_{+}$is the rank of a maximal positive definite subspace $H_{+}(X ; \mathbb{R})$ of $H_{2}(X ; \mathbb{R})$. His strategy for proof is to use the finite dimensional approximation introduced by M. Furuta [12] and appeal to equivariant $K$-theoretic devices such as the Adams $\psi$-operations. This method requires concrete informations about equivariant $K$ groups.

On the other hand, in this paper, we give an alternative proof of Fang's theorem by a completely different method which is rather geometric. Then we are able to extend it to the case when $b_{1} \geq 1$ by this geometric method.

To state the result, we need some preliminaries.

Let $G$ be the cyclic group of prime order $p$, and $X$ be a $G$-manifold. When $p=2$, we assume that the $G$-action is orientation-preserving. (Note that, when $p$ is odd, every $G$-action is orientation-preserving.) Fixing a $G$-invariant metric on $X$, we have a $G$-action on the frame bundle $P_{\text {SO }}$. According to [10], we say that a $\operatorname{Spin}^{c}$-structure $c$ is $G$-equivariant if the $G$-action on $P_{\mathrm{SO}}$ lifts to a $G$-action on the $\operatorname{Spin}^{c}(4)$-bundle $P_{\operatorname{Spin}^{c}}$ of $c$.

Suppose that a $G$-equivariant $\operatorname{Spin}^{c}$-structure $c$ is given. Fix a $G$-invariant connection $A_{0}$ on the determinant line bundle $L$ of $c$. Then the Dirac operator $D_{A_{0}}$ associated to $A_{0}$ is $G$-equivariant, and the $G$-index of $D_{A_{0}}$ can be written as $\operatorname{ind}_{G} D_{A_{0}}=\sum_{j=0}^{p-1} k_{j} \mathbb{C}_{j} \in R(G) \cong \mathbb{Z}[t] /\left(t^{p}-1\right)$, where $\mathbb{C}_{j}$ is the complex 1dimensional weight $j$ representation of $G$ and $R(G)$ is the representation ring of $G$.

For any $G$-space $V$, let $V^{G}$ be the fixed point set of the $G$-action. Let $b_{\bullet}^{G}=$ $\operatorname{dim} H_{\bullet}(X ; \mathbb{R})^{G}$, where $\bullet=1,2,+$. The Euler number of $X$ is denoted by $\chi(X)$, and the signature of $X$ by $\operatorname{Sign}(X)$.

In such a situation, F. Fang [10] proves the following theorem.

\footnotetext{
*Received September 13, 2005; accepted for publication February 23, 2006.

${ }^{\dagger}$ Research Institute for Mathematical Sciences, Kyoto university, Kyoto, 606-8502, Japan (nakamura@kurims.kyoto-u.ac.jp).
} 
THEOREM 1.1 ([10]). Let $G$ be the cyclic group of prime order $p$, and $X$ be a smooth closed oriented 4-dimensional $G$-manifold with $b_{1}=0$ and $b_{+} \geq 2$. Let $c$ be a $G$-equivariant $\mathrm{Spin}^{c}$-structure. Suppose $G$ acts on $H_{+}(X ; \mathbb{R})$ trivially. If $2 k_{j} \leq b_{+}-1$ for $j=0,1, \ldots, p-1$, then the Seiberg-Witten invariant $\mathrm{SW}_{X}(c)$ for $c$ satisfies

$$
\mathrm{SW}_{X}(c) \equiv 0 \quad \bmod p .
$$

We will generalize Theorem 1.1 to the case when $b_{1} \geq 1$. When $b_{1} \geq 1$, the whole theory can be viewed as a family on the Jacobian torus $J$. We consider the Jacobian torus $J$ as the set of equivalence classes of framed $\mathrm{U}(1)$-connections on $L$ whose curvatures are equal to that of the fixed $G$-invariant connection $A_{0}$. More concretely, $J$ is given as follows: Suppose that $X^{G} \neq \emptyset$, and choose a base point $x_{0} \in X^{G}$. Let $\mathcal{G}_{0}$ be the group of gauge transformations which are the identity at the base point $x_{0}$. Then the Jacobian $J$ is given as $J=\left(A_{0}+i \operatorname{ker} d\right) / \mathcal{G}_{0}$, where ker $d$ is the space of closed 1-forms. Note that $G$ acts on $J$, and $J$ is isomorphic to $H^{1}(X ; \mathbb{R}) / H^{1}(X ; \mathbb{Z}) G$-equivariantly.

Since $J$ as above gives a well-defined family of connections, we can also consider the family of Dirac operators $\left\{D_{A}\right\}_{[A] \in J}$. Then its $G$-index $\operatorname{ind}_{G}\left\{D_{A}\right\}_{[A] \in J}$ is an element of the $G$-equivariant $K$-group $K_{G}(J)$ over $J$.

Let $J^{G}=J_{0} \cup J_{1} \cup \cdots \cup J_{K}$ be the decomposition of the fixed point set $J^{G}$ into connected components. Choose a point $t_{l}$ in each $J_{l}$. For convenience, we assume that $J_{0}$ is the component including the origin which is represented by the fixed $G$-invariant connection $A_{0}$, and $t_{0}$ is the origin $\left[A_{0}\right]$. By restriction, we have homomorphisms $r_{l}: K_{G}(J) \rightarrow K_{G}\left(t_{l}\right)$. Since each $K_{G}\left(t_{l}\right)$ is just the representation ring $R(G) \cong \mathbb{Z}[t] /\left(t^{p}-1\right)$, the image of $\alpha=\operatorname{ind}_{G}\left\{D_{A}\right\}_{[A] \in J}$ by $r_{l}$ is written as $r_{l}(\alpha)=\sum_{j=0}^{p-1} k_{j}^{l} \mathbb{C}_{j}$. (When $X^{G}=\emptyset$, a well-defined $G$-equivariant family of connections can not be constructed in general. However coefficients $k_{j}^{l}$ can be defined ad hoc for our purpose. See §3.4.) Now we state our main result which is a generalization of Theorem 1.1.

TheOREm 1.2. Let $G$ be the cyclic group of prime order $p$, and $X$ be a smooth closed oriented 4-dimensional $G$-manifold with $b_{+} \geq 2$ and $b_{+}^{G} \geq 1$. Let $c$ be a $G$ equivariant $\operatorname{Spin}^{c}$-structure, and $L$ be the determinant line bundle of $c$. Suppose $d(c)=$ $\frac{1}{4}\left(c_{1}(L)^{2}-\operatorname{Sign}(X)\right)-\left(1-b_{1}+b_{+}\right)$is non-negative and even. If there exists a partition $\left(d_{0}, d_{1}, \ldots, d_{p-1}\right)$ of $d(c) / 2$ such that $d_{0}+d_{1}+\cdots+d_{p-1}=d(c) / 2$, and each $d_{j}$ is a non-negative integer and

$$
2 k_{j}^{l}<2 d_{j}+1-b_{1}^{G}+b_{+}^{G} \quad(\text { for } j=0,1, \ldots, p-1 \text { and any } l),
$$

then the Seiberg-Witten invariant $\mathrm{SW}_{X}(c)$ for c satisfies

$$
\mathrm{SW}_{X}(c) \equiv 0 \quad \bmod p .
$$

REMARK 1.4. The number $d(c)$ is the virtual dimension of the Seiberg-Witten moduli space $\mathcal{M}_{c}$ of $c$, and $\mathrm{SW}_{X}(c)$ denotes the Seiberg-Witten invariant which is defined by the formula $\mathrm{SW}_{X}(c)=\left\langle U^{\frac{d(c)}{2}},\left[\mathcal{M}_{c}\right]\right\rangle$, where $U$ is the cohomology class which comes from the U(1)-action. (See Definition 2.5 below.)

When $b_{1}>0$, we can evaluate the fundamental class $\left[\mathcal{M}_{c}\right]$ by cohomology classes which originate in the Jacobian torus $J$ and define corresponding invariants. Under 
our setting, there are some relations among these invariants which hold modulo $p$. This issue is treated separately in $\S 4$.

REMARK 1.5. It can be easily seen that Theorem 1.2 implies Theorem 1.1. By the assumption of Theorem 1.1, $b_{+}^{G}=b_{+} \geq 2$ and $b_{1}=b_{1}^{G}=0$. If $d(c)$ is odd or negative, then $\mathrm{SW}_{X}(c)=0$ by definition. Note that $d(c)$ is odd if and only if $b_{+}$is even. Therefore we can assume $d(c)$ is non-negative and $b_{+}$is odd. If the condition $2 k_{j} \leq b_{+}-1$ for any $j$ is satisfied, then (1.3) is satisfied for any partition of $d(c) / 2$. Therefore we obtain Theorem 1.1.

REMARK 1.6. Theorem 1.2 can be rewritten in the following simpler form: Let $X$ and $c$ be as in Theorem 1.2. Let $e_{j}$ (for $j=0, \ldots, p-1$ ) be integers defined by,

$$
e_{j}=\max _{l}\left\{\left(k_{j}^{l}-B\right), 0\right\}
$$

where the constant $B$ is given as

$$
B=\left\{\begin{array}{l}
\frac{1}{2}\left(1-b_{1}^{G}+b_{+}^{G}-1\right), \text { when } 1-b_{1}^{G}+b_{+}^{G} \text { is odd } \\
\frac{1}{2}\left(1-b_{1}^{G}+b_{+}^{G}-2\right), \text { when } 1-b_{1}^{G}+b_{+}^{G} \text { is even. }
\end{array}\right.
$$

If $\sum_{j=0}^{p-1} e_{j} \leq d(c) / 2$, then $\mathrm{SW}_{X}(c) \equiv 0 \bmod p$.

Let us consider more precisely about lifts of the $G$-action to a $\operatorname{Spin}^{c}$-structure. For a Spin ${ }^{c}$-structure $c$, we have a bundle map $P_{\mathrm{Spin}^{c}} \rightarrow P_{\mathrm{SO}} \times_{X} P_{\mathrm{U}(1)}$, where $P_{\mathrm{U}(1)}$ is the U(1) bundle for the determinant line bundle. This bundle map is a 2-fold covering. Suppose that $P_{\mathrm{U}(1)}$ is $G$-equivariant. If the action of a generator of $G$ on $P_{\mathrm{SO}} \times_{X} P_{\mathrm{U}(1)}$ lifts to $P_{\mathrm{Spin}^{c}}$, then all of such lifts form an action on $P_{\mathrm{Spin}^{c}}$ of an extension group $\hat{G}$ of $\mathbb{Z}_{2}$ by $G$ :

$$
1 \rightarrow \mathbb{Z}_{2} \rightarrow \hat{G} \rightarrow G \rightarrow 1
$$

When $G$ is an odd order cyclic group, (1.7) splits. Therefore, if $\hat{G}$-lifts exists,

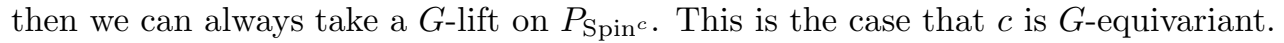

However, when $G=\mathbb{Z}_{2}$, (1.7) does not necessarily split. The non-split case is when $\hat{G}=\mathbb{Z}_{4}$. In such a case, we say that the $\mathbb{Z}_{2}$-action is of odd type with respect to $c$. On the other hand, when $c$ is $\mathbb{Z}_{2}$-equivariant, we say that the $\mathbb{Z}_{2}$-action is of even type with respect to $c$.

Now suppose that the $\mathbb{Z}_{2}$-action is of odd type with respect to $c$. For a $\mathbb{Z}_{2^{-}}$ connection $A$ on $L$, the Dirac operator $D_{A}$ is $\mathbb{Z}_{4}$-equivariant, and the $\mathbb{Z}_{4}$-index is of the form $\operatorname{ind}_{\mathbb{Z}_{4}} D_{A}=k_{1} \mathbb{C}_{1}+k_{3} \mathbb{C}_{3}$. (This is because the $\mathbb{Z}_{4}$-lift of the generator of $\mathbb{Z}_{2}$ acts on spinors as multiplication by $\pm \sqrt{-1}$.)

In this case, we also have a result similar to Theorem 1.2. (Compare with Theorem 2 in [10].)

THEOREM 1.8. Let $G=\mathbb{Z}_{2}$, and $X$ be a smooth closed oriented 4-dimensional $G$-manifold with $b_{+} \geq 2$ and $b_{+}^{G} \geq 1$. Suppose that the $G$-action is of odd type with respect to a $\operatorname{Spin}^{c}$-structure $c$. For such $(X, c)$, Theorem 1.2 holds as follows. If there exists a partition $\left(d_{1}, d_{3}\right)$ of $d(c) / 2$ such that $d_{1}+d_{3}=d(c) / 2$, and each $d_{j}$ is a non-negative integer and

$$
2 k_{j}^{l}<2 d_{j}+1-b_{1}^{G}+b_{+}^{G} \quad(\text { for } j=1,3 \text { and any } l),
$$


then the Seiberg-Witten invariant $\mathrm{SW}_{X}(c)$ for c satisfies

$$
\mathrm{SW}_{X}(c) \equiv 0 \quad \bmod 2 .
$$

Let us explain the outline of proofs of Theorem 1.2 and Theorem 1.8.

We also use a finite dimensional approximation $f$. We carry out the $G$-equivariant perturbation of $f$ to achieve the transversality, and then, under the assumption of (1.3), we see that the zero set of $f$ has no fixed point of the $G$-action by the dimensional reason concerning fixed point sets. Thus $G$ acts on the moduli space freely. Hence, if the dimension of moduli space is zero, then the number of elements in the moduli space is a multiple of $p$. From this, we can see that the Seiberg-Witten invariant is also a multiple of $p$. When the dimension of the moduli space is larger than 0 , it suffices to cut down the moduli space.

To conclude the introduction, let us give a remark. At present, we did not find an application of Theorem 1.2 in the case when $b_{1} \geq 1$. However, in the case of the $K 3$ surface whose $b_{1}$ is 0 , the author and X. Liu proved the existence of a locally linear action which can not be realized by a smooth action by using the mod $p$ vanishing theorem [14]. Therefore, we could use Theorem 1.2 or Theorem 1.8 to find such an action on a manifold with $b_{1} \geq 1$. This problem is left to the future research.

The paper is organized as follows: $\S 2$ gives a brief review on the finite dimensional approximation of the monopole map and Seiberg-Witten invariants in the $G$ equivariant setting. $\S 3$ proves Theorem 1.2 and Theorem 1.8. $\S 4$ deals with SeibergWitten invariants obtained from tori in the Jacobian. $\S 5$ gives some examples.

Acknowledgements. The author would like to express his deep gratitude to M. Furuta for invaluable discussions and continuous encouragements for years. It is also a pleasure to thank Y. Kametani for helpful discussions.

2. The $G$-equivariant finite dimensional approximation. The purpose of this section is to give a brief review on the finite dimensional approximation of the monopole map and Seiberg-Witten invariants in the $G$-equivariant setting.

2.1. The monopole map. Let $G=\mathbb{Z}_{p}$, where $p$ is prime, and $X$ be a smooth closed oriented 4-dimensional $G$-manifold with $b_{+} \geq 2$ and $b_{+}^{G} \geq 1$. Suppose that $X^{G} \neq \emptyset$.

Fix a $G$-invariant metric on $X$. Suppose a $\operatorname{Spin}^{c}$-structure $c$ is $G$-equivariant. We write $S^{+}$and $S^{-}$for the positive and negative spinor bundle of $c$. Let $L$ be the determinant line bundle: $L=\operatorname{det} S^{+}$.

The Seiberg-Witten equations are a system of equations for a U(1)-connection $A$ on $L$ and a positive spinor $\phi \in \Gamma\left(S^{+}\right)$,

$$
\left\{\begin{array}{c}
D_{A} \phi=0, \\
F_{A}^{+}=q(\phi),
\end{array}\right.
$$

where $D_{A}$ denotes the Dirac operator, $F_{A}^{+}$denotes the self-dual part of the curvature $F_{A}$, and $q(\phi)$ is the trace free part of the endomorphism $\phi \otimes \phi^{*}$ of $S^{+}$and this endomorphism is identified with an imaginary-valued self-dual 2-form via the Clifford multiplication.

The action of the gauge transformation group $\mathcal{G}=\operatorname{Map}(X ; \mathrm{U}(1))$ is given as follows: for $u \in \mathcal{G}, u(A, \phi)=\left(A-2 u^{-1} d u, u \phi\right)$. Let $\mathcal{M}_{c}$ denotes the moduli space of solutions,

$$
\mathcal{M}_{c}=\{\text { solutions to }(2.1)\} / \mathcal{G} \text {. }
$$


Fix a $G$-invariant connection $A_{0}$ on $L$. Choose a base point $x_{0}$ in $X^{G}$, and let $\mathcal{G}_{0}=\left\{u \in \mathcal{G} \mid u\left(x_{0}\right)=1\right\}$. Then $G$ acts on $\mathcal{G}_{0}$. The Jacobian torus $J$ is given as $J=\left(A_{0}+i \operatorname{Ker} d\right) / \mathcal{G}_{0}$, where $\operatorname{Ker} d$ is the space of closed 1-forms.

Let us define infinite dimensional bundles $\mathcal{V}$ and $\mathcal{W}$ over $J$ by

$$
\begin{aligned}
\mathcal{V} & =\left(A_{0}+i \operatorname{Ker} d\right) \times_{\mathcal{G}_{0}}\left(\Gamma\left(S^{+}\right) \oplus \Omega^{1}(X)\right), \\
\mathcal{W} & =\left(A_{0}+i \operatorname{Ker} d\right) \times_{\mathcal{G}_{0}}\left(\Gamma\left(S^{-}\right) \oplus \Omega^{+}(X) \oplus H^{1}(X ; \mathbb{R}) \oplus \Omega^{0}(X) / \mathbb{R}\right),
\end{aligned}
$$

where $\mathbb{R}$ is the space of constant functions and $\mathcal{G}_{0}$-actions on spaces of forms and $H^{1}(X ; \mathbb{R})$ are trivial. Note that $\mathcal{V}$ decomposes into $\mathcal{V}=\mathcal{V}_{\mathbb{C}} \oplus \mathcal{V}_{\mathbb{R}}$, where $\mathcal{V}_{\mathbb{C}}$ is a complex bundle come from the component $\Gamma\left(S^{+}\right)$on which $\mathrm{U}(1)$ acts by weight 1 , and $\mathcal{V}_{\mathbb{R}}$ is a real bundle come from $\Omega^{1}(X)$ on which $\mathrm{U}(1)$ acts trivially. The bundle $\mathcal{W}$ decomposes similarly as $\mathcal{W}=\mathcal{W}_{\mathbb{C}} \oplus \mathcal{W}_{\mathbb{R}}$.

To carry out appropriate analysis, we have to complete these spaces with suitable Sobolev norms. Fix an integer $k>4$, and take the fiberwise $L_{k}^{2}$-completion of $\mathcal{V}$ and the fiberwise $L_{k-1}^{2}$-completion of $\mathcal{W}$. For simplicity, we use the same notation for completed spaces.

Now we define the monopole map $\Psi: \mathcal{V} \rightarrow \mathcal{W}$ by

$$
\Psi(A, \phi, a)=\left(A, D_{A+i a} \phi, F_{A+i a}^{+}-q(\phi), h(a), d^{*} a\right)
$$

where $h(a)$ denotes the harmonic part of the 1-form $a$. In our setting, $\Psi$ is a $\mathrm{U}(1) \times G$ equivariant bundle map. Note that the moduli space $\mathcal{M}_{c}$ exactly coincides with $\Psi^{-1}(0) / \mathrm{U}(1)$.

2.2. Finite dimensional approximation. In this subsection, we describe the finite dimensional approximation of the monopole map according to [13]. (See also [6].)

Decompose the monopole map $\Psi$ into the sum of linear part $\mathcal{D}$ and quadratic part $\mathcal{Q}$, i.e., $\Psi=\mathcal{D}+\mathcal{Q}$, where $\mathcal{D}: \mathcal{V} \rightarrow \mathcal{W}$ is given by

$$
\mathcal{D}(A, \phi, a)=\left(A, D_{A} \phi, d^{+} a, h(a), d^{*} a\right),
$$

and $\mathcal{Q}$ is the rest.

Let $W_{\lambda}$ (resp. $V_{\lambda}$ ) be the subspace of $\mathcal{W}$ (resp. $\mathcal{V}$ ) spanned by eigenspaces of $\mathcal{D D}^{*}\left(\right.$ resp. $\left.\mathcal{D}^{*} \mathcal{D}\right)$ with eigenvalues less than or equal to $\lambda$. Let $p_{\lambda}: \mathcal{W} \rightarrow W_{\lambda}$ be the orthogonal projection. As in [12], we would like to consider $\mathcal{D}+p_{\lambda} \mathcal{Q}$ as a finite dimensional approximation of $D+\mathcal{Q}$. However $W_{\lambda}$ and $p_{\lambda}$ do not vary continuously with respect to parameters in $J$. It is necessary to modify these.

Let $\beta:(-1,0) \rightarrow[0, \infty)$ be a compact-supported smooth non-negative cut-off function whose integral over $(-1,0)$ is 1 . For each $\lambda>1$, let us define the smoothing of the projection $\tilde{p}_{\lambda}: \mathcal{W} \rightarrow W_{\lambda}$ by

$$
\int_{-1}^{0} \beta(t) p_{\lambda+t} d t
$$

Let $\iota_{\lambda}: W_{\lambda} \rightarrow \mathcal{W}$ be the inclusion. Then the composition $\iota_{\lambda} \tilde{p}_{\lambda}$ varies continuously.

For a fixed $\lambda$, we replace $W_{\lambda}$ with a vector bundle $W_{f}$ in the following lemma.

Lemma 2.2 (See [13]). There is a $\mathrm{U}(1) \times G$-equivariant finite-rank vector bundle $W_{f}$ over $J$ and $\mathrm{U}(1) \times G$-equivariant bundle homomorphisms $\chi: W_{f} \rightarrow \mathcal{W}$ and $s: \mathcal{W} \rightarrow W_{f}$ which have the following properties. 
(1) The composition $\chi s$ on $W_{\lambda}$ is the identity. In particular, the image of $\chi$ contains $W_{\lambda}$.

(2) There is a $\mathrm{U}(1) \times G$-equivariant isomorphism from $W_{f}$ to the product bundle $J \times F_{\mathbb{C}} \oplus F_{\mathbb{R}}$, where $F_{\mathbb{C}}$ and $F_{\mathbb{R}}$ are complex and real representations of $G$ respectively.

The proof of Lemma 2.2 is given by modifying the proof of Lemma 3.2 in [13] $G$-equivariantly.

Let us consider the map $\mathcal{D}+\chi: \mathcal{V} \oplus W_{f} \rightarrow \mathcal{W}$. Then we can show from Lemma 2.2 that this map is always surjective. Therefore $V_{f}:=\operatorname{Ker}(\mathcal{D}+\chi)$ becomes a $\mathrm{U}(1) \times G$ equivariant finite-rank vector bundle.

Now we can replace the family of linear maps $\mathcal{D}: V_{\lambda} \rightarrow W_{\lambda}$ with

$$
\mathcal{D}_{f}: V_{f} \rightarrow W_{f}, \quad(v, e) \mapsto e,
$$

which depends continuously on the parameter space $J$. Note that the formal difference $\left[V_{f}\right]-\left[W_{f}\right]$ gives the index of family $\mathcal{D}: V_{\lambda} \rightarrow W_{\lambda}$. In fact, it is easy to see that $\operatorname{ker} \mathcal{D} \cong \operatorname{ker} \mathcal{D}_{f}$ and coker $\mathcal{D} \cong \operatorname{coker} \mathcal{D}_{f}$.

For the non-linear part $\mathcal{Q}$, we define a continuous family $\mathcal{Q}_{f}: V_{f} \rightarrow W_{f}$ by

$$
\mathcal{Q}_{f}(v, e)=-s \iota_{\lambda} \tilde{p}_{\lambda} \mathcal{Q}(v) .
$$

Then the map $\Psi_{f}:=\mathcal{D}_{f}+\mathcal{Q}_{f}$ gives a finite dimensional approximation of $\Psi=$ $\mathcal{D}+\mathcal{Q}$ when we take sufficiently large $\lambda$. This is a $\mathrm{U}(1) \times G$-equivariant and proper map. In particular, the inverse image of zero is compact.

REMARK 2.3. The formulation in [6] is simpler than that of this section or [13]. However we need to use this formulation because the method in [6] requires a trivialization of $\mathcal{W}$. In the non-equivariant setting, $\mathcal{W}$ can be always trivialized by Kuiper's theorem. However, in the $G$-equivariant setting, we do not know whether $\mathcal{W}$ can be trivialized $G$-equivariantly, or not.

2.3. Seiberg-Witten invariants. Let $f_{0}=\Psi_{f}: V \rightarrow W$ be a finite dimensional approximation. The space $V$ decomposes into the sum of a complex vector bundle $V_{\mathbb{C}}$ and a real vector bundle $V_{\mathbb{R}}, V=V_{\mathbb{C}} \oplus V_{\mathbb{R}}$, according to the splitting $\mathcal{V}=\mathcal{V}_{\mathbb{C}} \oplus \mathcal{V}_{\mathbb{R}}$. Similarly $W=W_{\mathbb{C}} \oplus W_{\mathbb{R}}$. Note that $\left[V_{\mathbb{C}}\right]-\left[W_{\mathbb{C}}\right]$ gives the $G$-index of the family of Dirac operators $\left\{D_{A}\right\}_{[A] \in J}$. Note also that $V_{\mathbb{R}}$ is a trivial bundle $\underline{F}=J \times F$, where $F$ is a real representation of $G$, and $W_{\mathbb{R}}=\underline{F} \oplus \underline{H}^{+}$, where $\underline{\underline{H}}^{+}=J \times H^{+}(X ; \mathbb{R})$.

To obtain the Seiberg-Witten invariant, we need to perturb $f_{0}$ in general. For our purpose, we need to carry out the perturbation $G$-equivariantly. First, note that the moduli space $\mathcal{M}_{c}=f_{0}^{-1}(0) / \mathrm{U}(1)$ may have $\mathrm{U}(1)$-quotient singularities. (They are called reducibles. Strictly speaking, $f_{0}^{-1}(0) / \mathrm{U}(1)$ does not coincide with the genuine moduli space of solutions in general. However, after perturbation, the fundamental class of $f_{0}^{-1}(0) / \mathrm{U}(1)$ is equal to that of the perturbed moduli space. Therefore we abuse the term "moduli space" and the notation $\mathcal{M}_{c}$ for $f_{0}^{-1}(0) / \mathrm{U}(1)$.) Let us consider the restriction of $f_{0}$ to the $\mathrm{U}(1)$-invariant part of $V$. The $\mathrm{U}(1)$-invariant parts of $V$ and $W$ are $V^{\mathrm{U}(1)}=V_{\mathbb{R}}=\underline{F}$, and $W^{\mathrm{U}(1)}=W_{\mathbb{R}}=\underline{F} \oplus \underline{H}^{+}$, respectively. Since the restriction $\left.f_{0}\right|_{V^{U}(1)}$ is a fiberwise linear proper map, this is just a fiberwise linear inclusion. Therefore, by fixing a non-zero vector $v \in H^{+}(X ; \mathbb{R})^{G} \backslash\{0\}$, and perturbing $f_{0}$ to $f=f_{0}+v$, we can avoid reducibles, that is, $f^{-1}(0)^{\mathrm{U}(1)}=\emptyset$. (Note that this perturbation is $\mathrm{U}(1) \times G$-equivariant.) 
Let $\bar{V}=\left(\left(V_{\mathbb{C}} \backslash\{0\}\right) \times_{J} V_{\mathbb{R}}\right) / \mathrm{U}(1)$, and define a vector bundle $\bar{E} \rightarrow \bar{V}$ by

$$
\bar{E}=\left(\left(V_{\mathbb{C}} \backslash\{0\}\right) \times_{J} V_{\mathbb{R}} \times_{J} W\right) / \mathrm{U}(1) .
$$

Since $f$ is U(1)-equivariant, $f$ induces a section $\bar{f}: \bar{V} \rightarrow \bar{E}$. Now, the moduli space $\mathcal{M}_{c}$ is the zero locus of $\bar{f}$. Suppose $\bar{f}$ is transverse to the zero section of $\bar{E}$. (In general, we need a second perturbation. Furthermore, in our case, the perturbation should be $G$-equivariant. This is a task in $\S 3$.) Then the moduli space $\mathcal{M}_{c}=\bar{f}^{-1}(0)$ becomes a compact manifold whose dimension $d(c)$ is

$$
d(c)=\frac{1}{4}\left(c_{1}(L)^{2}-\operatorname{Sign}(X)\right)-\left(1-b_{1}+b_{+}\right) .
$$

We can determine the orientation of $\mathcal{M}_{c}$ from an orientation of $H^{1}(X ; \mathbb{R}) \oplus H^{+}(X ; \mathbb{R})$.

Let us introduce a complex line bundle $\mathcal{L} \rightarrow \bar{V}$ by $\mathcal{L}=\left(\left(V_{\mathbb{C}} \backslash\{0\}\right) \times_{J} V_{\mathbb{R}}\right) \times_{\mathrm{U}(1)} \mathbb{C}$, where $\mathrm{U}(1)$ action on $\mathbb{C}$ is multiplication. Let $U=c_{1}(\mathcal{L})$. Note that $H^{*}(\bar{V} ; \mathbb{Z})$ is isomorphic to $\mathbb{Z}[U] /\left(U^{D}-1\right) \otimes H^{*}(J ; \mathbb{Z})$ for some $D$ as an additive group.

Now we give the definition of the Seiberg-Witten invariants.

Definition 2.5. The Seiberg-Witten invariant for a $\operatorname{Spin}^{c}$-structure $c$ is given as a map,

$$
\mathrm{SW}_{X, c}: \mathbb{Z}[U] \otimes H^{*}(J ; \mathbb{Z}) \rightarrow \mathbb{Z}
$$

which is defined by $\mathrm{SW}_{X, c}\left(U^{d} \otimes \xi\right)=\left\langle U^{d} \cup \xi,\left[\mathcal{M}_{c}\right]\right\rangle$.

Note that an element $\xi$ in $H^{*}(J ; \mathbb{Z})$ can be written as a linear combination of Poincare duals of homology classes represented by subtori in $J$.

Let $T$ be a subtorus in $J$, and its dimension be $d_{T}$. Suppose $d(c)-d_{T}$ is even and non-negative. Put $d^{\prime}=\left(d(c)-d_{T}\right) / 2$. Then the Seiberg-Witten invariant $\mathrm{SW}_{X, c}\left(U^{d^{\prime}} \otimes\right.$ P.D.[T]) can be represented geometrically as follows: Let $\mathcal{L}_{1}, \mathcal{L}_{2}, \ldots, \mathcal{L}_{d^{\prime}}$ be $d^{\prime}$ copies of $\mathcal{L}$ and $s_{i}: \bar{V} \rightarrow \mathcal{L}_{i}\left(i=1,2, \ldots, d^{\prime}\right)$ be arbitrary sections. Consider a section $\bar{f}_{C}$ of the vector bundle $\bar{E} \oplus \mathcal{L}_{1} \oplus \cdots \oplus \mathcal{L}_{d^{\prime}}$ given by $\bar{f}_{C}=\left(\bar{f}, s_{1}, \ldots, s_{d^{\prime}}\right)$. Now restrict $\bar{f}_{C}$ to $\left.\bar{V}\right|_{T}$. If $\left.\bar{f}_{C}\right|_{\left.\bar{V}\right|_{T}}$ is transverse to the zero section, then $\mathrm{SW}_{X, c}\left(U^{d^{\prime}} \otimes P . D .[T]\right)$ is equal to the signed count of zeros of $\left.\bar{f}_{C}\right|_{\left.\bar{V}\right|_{T}}$ according to their orientations. (This method is called cutting down the moduli space.)

In this paper, we use the notation

$$
\mathrm{SW}_{X}(c)=\mathrm{SW}_{X, c}\left(U^{\frac{d(c)}{2}}\right),
$$

when $d(c)$ is non-negative and even.

3. G-equivariant perturbation of $\bar{f}$. In this section, we carry out the $G$ equivariant perturbation of $\bar{f}$, and finally prove Theorem 1.2 and Theorem 1.8.

Up to this point, we obtained a $G$-equivariant section $\bar{f}: \bar{V} \rightarrow \bar{E}$ which have no $\mathrm{U}(1)$-quotient singularity in the zero locus. That is, the moduli space contains no reducible. In order to go further, we need to identify $G$-fixed point sets $\bar{V}^{G}$ and $\bar{E}^{G}$.

3.1. Fixed point sets $\bar{V}^{G}$ and $\bar{E}^{G}$. Let us summarize the notation so far. The (perturbed) finite dimensional approximation is

$$
f: V=V_{\mathbb{C}} \oplus \underline{F} \rightarrow W=W_{\mathbb{C}} \oplus \underline{F} \oplus \underline{H}^{+} .
$$

The induced section is

$$
\bar{f}: \bar{V}=\left(V_{\mathbb{C}} \backslash\{0\}\right) / \mathrm{U}(1) \times_{J} \underline{F} \rightarrow \bar{E}=\left(\left(V_{\mathbb{C}} \backslash\{0\}\right) \times_{J} W_{\mathbb{C}}\right) / \mathrm{U}(1) \times_{J}\left(\underline{F} \oplus \underline{F} \oplus \underline{H}^{+}\right) .
$$


Let us identify the fixed point set $\bar{V}^{G}=\left(\left(V_{\mathbb{C}} \backslash\{0\}\right) / \mathrm{U}(1) \times_{J} \underline{F}\right)^{G}$. Note that $\bar{V}^{G} \rightarrow J^{G}$ is a fiber bundle. Recall that $\left[V_{\mathbb{C}}\right]-\left[W_{\mathbb{C}}\right]=\operatorname{ind}_{G}\left\{D_{A}\right\}_{[A] \in J}$. Then, for a fixed point $t_{l} \in J_{l} \subset J^{G}$, fibers of $V_{\mathbb{C}}$ and $W_{\mathbb{C}}$ over $t_{l}$ are written as

$$
\left.V_{\mathbb{C}}\right|_{t_{l}}=\sum_{j=0}^{p-1} k_{j}^{l+} \mathbb{C}_{j},\left.\quad W_{\mathbb{C}}\right|_{t_{l}}=\sum_{j=0}^{p-1} k_{j}^{l-} \mathbb{C}_{j},
$$

and the relation $k_{j}^{l}=k_{j}^{l+}-k_{j}^{l-}$ holds. Therefore the fiber of $\bar{V}^{G}$ over $t_{l}$ is $\left.\bar{V}^{G}\right|_{t_{l}}=\left(\left(\sum_{j=0}^{p-1} k_{j}^{l+} \mathbb{C}_{j} \backslash\{0\}\right) / \mathrm{U}(1)\right)^{G} \times F_{0}$, where $F_{0}$ is the $G$-invariant part of the real representation $F$.

LEMma 3.1. There is a homeomorphism

$$
\left(\left(\sum_{j=0}^{p-1} k_{j}^{l+} \mathbb{C}_{j} \backslash\{0\}\right) / \mathrm{U}(1)\right)^{G} \cong \coprod_{j=0}^{p-1} P\left(k_{j}^{l+} \mathbb{C}_{j}\right) \times \mathbb{R}_{+},
$$

where $P\left(k_{j}^{l+} \mathbb{C}_{j}\right)$ is the projective space of $k_{j}^{l+} \mathbb{C}_{j}$, and $\mathbb{R}_{+}$is the set of positive real numbers.

Proof. Note that there is a $G$-equivariant homeomorphism

$$
\left(\sum_{j=0}^{p-1} k_{j}^{l+} \mathbb{C}_{j} \backslash\{0\}\right) / \mathrm{U}(1) \cong P\left(\sum_{j=0}^{p-1} k_{j}^{l+} \mathbb{C}_{j}\right) \times \mathbb{R}_{+} .
$$

A point $v$ in $P\left(\sum_{j=0}^{p-1} k_{j}^{l+} \mathbb{C}_{j}\right)$ is represented by a vector $\left(v_{0}, \ldots, v_{p-1}\right)$ where $v_{j} \in$ $k_{j}^{l+} \mathbb{C}_{j}$. Let $\zeta=\exp (2 \pi \sqrt{-1} / p)$. A point $v$ is fixed by the $G$-action if and only if there exists $\lambda \in \mathbb{C} \backslash\{0\}$ which satisfies $\lambda v_{j}=\zeta^{j} v_{j}$ for all $j$. Therefore there is a unique $j$ such that $v_{j} \neq 0$, and we have $\lambda=\zeta^{j}$ and $v_{j^{\prime}}=0$ for all $j^{\prime} \neq j$. Thus the lemma holds. $\mathrm{Q}$

By Lemma 3.1, we see that $\left.\bar{V}^{G}\right|_{t_{l}} \cong \coprod_{j=0}^{p-1} P\left(k_{j}^{l+} \mathbb{C}_{j}\right) \times \mathbb{R}_{+} \times F_{0}$. Therefore the dimension of the component $\bar{V}_{l, j}^{G}$ of $\bar{V}^{G}$ is given by

$$
\operatorname{dim} \bar{V}_{l, j}^{G}=2 k_{j}^{l+}-1+a+b_{1}^{G},
$$

where $\bar{V}_{l, j}^{G}$ denotes the $j$-th component over $J_{l} \subset J^{G}$, and $a=\operatorname{rank} F_{0}$. (Note that $b_{1}^{G}$ is the dimension of the base space $J_{l}$.)

Let us identify the fixed point set $\bar{E}^{G}$ similarly. Note that

$$
\bar{E}=\left(\left(V_{\mathbb{C}} \backslash\{0\}\right) \times_{J} W_{\mathbb{C}}\right) / \mathrm{U}(1) \times_{J}\left(\underline{F} \oplus \underline{F} \oplus \underline{H}^{+}\right)
$$

is an open submanifold of

$$
\bar{E}^{\prime}:=\left(\left(V_{\mathbb{C}} \oplus W_{\mathbb{C}}\right) \backslash\{0\}\right) / \mathrm{U}(1) \times_{J}\left(\underline{F} \oplus \underline{F} \oplus \underline{H}^{+}\right) .
$$

By the method similar to Lemma 3.1 , we see that $\left.\bar{E}^{\prime G}\right|_{t_{l}} \cong \coprod_{j=0}^{p-1} P\left(\left(k_{j}^{l+}+k_{j}^{l-}\right) \mathbb{C}_{j}\right) \times$ $\mathbb{R}_{+} \times\left(F_{0} \oplus F_{0} \oplus\left(H^{+}\right)^{G}\right)$. Therefore the dimension of the component $\bar{E}_{l, j}^{G}$ of $\bar{E}^{G}$ is given by

$$
\operatorname{dim} \bar{E}_{l, j}^{G}=2\left(k_{j}^{l+}+k_{j}^{l-}\right)-1+2 a+b_{+}^{G}+b_{1}^{G},
$$


where $\bar{E}_{l, j}^{G}$ denotes the $j$-th component over $J_{l} \subset J^{G}$.

Note that $\bar{E}^{G} \rightarrow \bar{V}^{G}$ is the disjoint union of vector bundles $\bar{E}_{l, j}^{G} \rightarrow \bar{V}_{l, j}^{G}$. The rank of $\bar{E}_{l, j}^{G}$ is given by

$$
\operatorname{rank}_{\mathbb{R}} \bar{E}_{l, j}^{G}=\operatorname{dim} \bar{E}_{l, j}^{G}-\operatorname{dim} \bar{V}_{l, j}^{G}=2 k_{j}^{l-}+a+b_{+}^{G} .
$$

3.2. Proof of Theorem 1.2 in the case when $d(c)=0$. Suppose now that $d(c)=0$. Under the assumption (1.3), formulae (3.2) and (3.3) imply that

$$
\operatorname{dim} \bar{V}_{l, j}^{G}<\operatorname{rank}_{\mathbb{R}} \bar{E}_{l, j}^{G} .
$$

Therefore, we can perturb the section $\bar{f}: \bar{V} \rightarrow \bar{E}$ on a small neighborhood of the fixed point set $\bar{V}^{G} G$-equivariantly so that $\bar{f}$ has no zero on $\bar{V}^{G}$. Then it is easy to carry out a $G$-equivariant perturbation outside the $G$-fixed point sets so that $\bar{f}$ is transverse to the zero section. (For instance, consider on quotient spaces $\bar{V} / G$ and $\bar{E} / G$, and then pull back to original spaces.)

Note that the moduli space $\mathcal{M}_{c}=\bar{f}^{-1}(0)$ no longer contains any $G$-fixed point. Hence $G$ acts freely on $\mathcal{M}_{c}$. Thus we have $\mathrm{SW}_{X}(c) \equiv 0 \bmod p$.

3.3. Proof of Theorem 1.2 in the case when $d(c)$ is positive and even. Let us introduce $G$-equivariant complex line bundles $\mathcal{L}_{j}$ over $\bar{V}(j=0, \ldots, p-1)$ by

$$
\mathcal{L}_{j}=\left(\left(V_{\mathbb{C}} \backslash\{0\}\right) \times_{J} V_{\mathbb{R}}\right) \times_{\mathrm{U}(1)} \mathbb{C}_{j},
$$

and fix $G$-equivariant sections $s_{j}: \bar{V} \rightarrow \mathcal{L}_{j}$. (It is easy to make a $G$-equivariant section. Choose an arbitrary non- $G$-equivariant section, and average it by the $G$-action.) We will cut down the moduli space by these $\left(\mathcal{L}_{j}, s_{j}\right)$.

Fix a partition $\left(d_{0}, d_{1}, \ldots, d_{p-1}\right)$ of $d(c) / 2$ such that $d_{j} \geq 0$ and $d_{0}+d_{1}+\cdots+$ $d_{p-1}=d(c) / 2$. Instead of the section $\bar{f}: \bar{V} \rightarrow \bar{E}$, we consider

$$
\bar{f}_{C}: \bar{V} \rightarrow \bar{E} \oplus d_{0} \mathcal{L}_{0} \oplus \cdots \oplus d_{p-1} \mathcal{L}_{p-1}=: \bar{E}_{C}
$$

which is defined by

$$
\bar{f}_{C}=\left(\bar{f}, s_{0}, \ldots, s_{0}, s_{1}, \ldots, s_{p-1}\right) .
$$

Hereafter, we argue in analogous way to that of $\S 3.1$. We write $\left(\bar{E}_{C}\right)_{l, j}^{G}$ for the component of the fixed point set $\left(\bar{E}_{C}\right)^{G}$ over $\bar{V}_{l, j}^{G}$. Then the rank of the vector bundle $\left(\bar{E}_{C}\right)_{l, j}^{G} \rightarrow \bar{V}_{l, j}$ is given by

$$
\operatorname{rank}_{\mathbb{R}}\left(\bar{E}_{C}\right)_{l, j}^{G}=2\left(k_{j}^{l-}+d_{j}\right)+a+b_{+}^{G} .
$$

An argument similar to that of the case when $d(c)=0$ in $\S 3.2$ completes the proof of Theorem 1.2 when $X^{G} \neq \emptyset$.

3.4. The case when $X^{G}=\emptyset$. The base point $x_{0} \in X^{G}$ is used for the welldefined $G$-equivariant family of connections over the Jacobian $J$. When $b_{1}=0$, we do not need the base point to construct a finite dimensional approximation. Therefore, the argument in this section also works in the case when $b_{1}=0$ and $X^{G}=\emptyset$. On the other hand, in the case when $b_{1} \geq 1$ and $X^{G}=\emptyset$, we can define coefficients $k_{j}^{l}$ ad hoc for our purpose, although we do not have a well-defined $G$-equivariant family of connections. Consider the Jacobian $J$ as $J=\left(A_{0}+i \operatorname{ker} d\right) / \mathcal{G}$, where $\mathcal{G}$ is the 
full gauge transformation group. Decompose the $G$-fixed point set $J^{G}$ into connected components: $J^{G}=J_{0} \cup \cdots \cup J_{K}$. Choose a point $t_{l}$ in each component $J_{l}$ and a connection $A_{l}$ in each class $t_{l}$. We assume that $J_{0}$ is the component of $\left[A_{0}\right]$ and $t_{0}=\left[A_{0}\right]$, where $A_{0}$ is the fixed $G$-equivariant connection. Then, for each $A_{l}$, we can redefine the $G$-action on the $\operatorname{Spin}^{c}$-structure $c$ such that $A_{l}$ is fixed by the redefined $G$-action. (This is proved as in Lemma 5.4.) Then the Dirac operator $D_{A_{l}}$ is $G$ equivariant, and the $G$-index $\operatorname{ind}_{G} D_{A_{l}}$ is written as $\operatorname{ind}_{G} D_{A_{l}}=\sum_{j=0}^{p-1} k_{j}^{l} \mathbb{C}_{j}$. In such a situation, we can prove the following.

Lemma 3.5. Suppose that $d(c)$ in (2.4) is nonnegative and even. If $X^{G}=\emptyset$, then there is no partition $\left(d_{0}, d_{1}, \ldots, d_{p-1}\right)$ of $d(c) / 2$ which satisfies (1.3).

Proof. Coefficients $k_{j}^{l}$ are calculated by the $G$-index theorem. (See $\S 5.1$.) In fact, we can show that

$$
k_{0}^{l}=k_{1}^{l}=\cdots=k_{p-1}^{l}=\frac{1}{p} \text { ind } D_{A_{0}}=\frac{1}{8 p}\left(c_{1}(L)^{2}-\operatorname{Sign}(X)\right),
$$

for any $l$. Note that $1-b_{1}+b_{+}=p\left(1-b_{1}^{G}+b_{+}^{G}\right)$ when $X^{G}=\emptyset$. (This follows from the formulae $\chi(X)=p \chi(X / G)$ and $\operatorname{Sign}(X)=p \operatorname{Sign}(X / G)$.) Therefore (1.3) is equivalent to $\frac{1}{p} d(c)<2 d_{j}$ for $j=0,1, \ldots, p-1$. Summing up these equations from $j=0$ to $p-1$ implies a contradiction. $\mathrm{Q}$

Therefore, the assumption $X^{G} \neq \emptyset$ can be omitted logically.

3.5. Proof of Theorem 1.8. Let $G=K=\mathbb{Z}_{2}$ and $\hat{G}=\mathbb{Z}_{4}$, and consider the short exact sequence,

$$
0 \rightarrow K \rightarrow \hat{G} \rightarrow G \rightarrow 0 .
$$

If the $G$-action is of odd type with respect to a $\operatorname{Spin}^{c}$-structure $c$, then $\hat{G}$ acts on the whole theory. In this case also, as in $\S 2$, we obtain the $\mathrm{U}(1) \times \hat{G}$-equivariant finite dimensional approximation

$$
f: V=V_{\mathbb{C}} \oplus \underline{F} \rightarrow W=W_{\mathbb{C}} \oplus \underline{F} \oplus \underline{H}^{+} .
$$

Note that the $\hat{G}$-action on $J, \underline{F}$ and $\underline{H}^{+}$factors through the surjection $\hat{G} \rightarrow G$, and hence the actions of the subgroup $K \subset \hat{G}$ on $J, \underline{F}$ and $\underline{H}^{+}$are trivial.

We need to identify $K$-fixed point sets as well as $\hat{G}$-fixed point sets. Note that $K$-actions on $V_{\mathbb{C}}$ and $W_{\mathbb{C}}$ are given as multiplication by -1 on each fiber, which are absorbed by $\mathrm{U}(1)$-actions. Therefore $K$-actions on $\bar{V}$ and $\bar{E}$ are trivial.

Thus we see that the $\hat{G}$-action on the section $\bar{f}: \bar{V} \rightarrow \bar{E}$ is reduced to an action of $G=\hat{G} / K$. Then, an argument analogous to $\S 3.1, \S 3.2, \S 3.3$ and $\S 3.4$ proves Theorem 1.8.

4. Cutting down the moduli by tori in $J$. This section deals with SeibergWitten invariants obtained from tori in $J$. In this section, let $G=\mathbb{Z}_{p}$ where $p$ is prime, and suppose that $X$ is a closed oriented 4 -dimensional $G$-manifold with $b_{+} \geq 2, b_{+}^{G} \geq 1$ and $b_{1} \geq 1$, and $X^{G} \neq \emptyset$. Let $c$ be a $G$-equivariant $\operatorname{Spin}^{c}$-structure.

First, we suppose that a subtorus $T$ in $J$ is $G$-invariant, i.e., $T=g T$ for $g \in$ $G$. Let $d_{T}=\operatorname{dim} T$. Suppose that $d(c)-d_{T}$ is non-negative and even, and put $d^{\prime}=\frac{1}{2}\left(d(c)-d_{T}\right)$. For a partition $\left(d_{0}, d_{1}, \ldots, d_{p-1}\right)$ of $d^{\prime}$, consider $\bar{f}_{C}: \bar{V} \rightarrow \bar{E}_{C}=$ $\bar{E} \oplus d_{0} \mathcal{L}_{0} \oplus \cdots \oplus d_{p-1} \mathcal{L}_{p-1}$ as in $\S 3.3$. Then consider the restriction $\left.\bar{f}_{C}\right|_{\left.\bar{V}\right|_{T}}$ of $\bar{f}_{C}$ to $\left.\bar{V}\right|_{T}$. By perturbing $\left.\bar{f}_{C}\right|_{\left.\bar{V}\right|_{T}} G$-equivariantly in the way similar to that of $\S 3$, we can prove the following. 
Theorem 4.1. Let $d_{T}^{G}=\operatorname{dim} T^{G}$. Suppose that $X^{G} \neq \emptyset$ and that $d(c)-d_{T}$ is nonnegative and even. Put $d^{\prime}=\frac{1}{2}\left(d(c)-d_{T}\right)$. If there exist a partition $\left(d_{0}, d_{1}, \ldots, d_{p-1}\right)$ of $d^{\prime}$ such that $d_{0}+d_{1}+\cdots+d_{p-1}=d^{\prime}$, and each $d_{j}$ is a non-negative integer and

$$
2 k_{j}^{l}<2 d_{j}+1-d_{T}^{G}+b_{+}^{G} \quad(\text { for } j=0,1, \ldots, p-1 \text { and any } l),
$$

then

$$
\mathrm{SW}_{X, c}\left(U^{d^{\prime}} \otimes P . D .[T]\right) \equiv 0 \quad \bmod p,
$$

where $k_{j}^{l}$ are defined similarly from $\operatorname{ind}_{G}\left\{D_{A}\right\}_{[A] \in T} \in K_{G}(T)$.

On the other hand, when $T$ is not $G$-invariant, the following holds.

Theorem 4.2. Let $d_{T}^{G}=\operatorname{dim} T^{G}$. Suppose that $X^{G} \neq \emptyset$ and that $d(c)-d_{T}$ is nonnegative and even. Put $d^{\prime}=\frac{1}{2}\left(d(c)-d_{T}\right)$. If there exist a partition $\left(d_{0}, d_{1}, \ldots, d_{p-1}\right)$ of $d^{\prime}$ such that $d_{0}+d_{1}+\cdots+d_{p-1}=d^{\prime}$, and each $d_{j}$ is a non-negative integer and

$$
2 k_{j}^{l}<2 d_{j}+1-d_{T}^{G}+b_{+}^{G} \quad(\text { for } j=1,2, \ldots, p-1 \text { and any } l),
$$

then

$$
\sum_{i=0}^{p-1} \mathrm{SW}_{X, c}\left(U^{d^{\prime}} \otimes P . D \cdot\left[g^{i} T\right]\right) \equiv 0 \quad \bmod p .
$$

Proof. Let us consider $\tilde{T}=T \cup g T \cup g^{2} T \cup \cdots \cup g^{p-1} T$ for $g \in G$, and the restriction $\left.\bar{f}_{C}\right|_{\left.\bar{V}\right|_{\tilde{T}}}$ of $f_{C}$ to $\left.\bar{V}\right|_{\tilde{T}}$. Note that $\tilde{T}$ is not necessarily a manifold. Let $T_{k}$ be the set of $t \in \tilde{T}$ such that the number of $g^{i} T(i=0,1, \ldots, p-1)$ which contains $t$ is lager than or equal to $k$, that is,

$$
T_{k}=\left\{t \in \tilde{T} \mid \#\left\{i \mid t \in g^{i} T\right\} \geq k\right\} .
$$

Note that $T_{1}=\tilde{T}$ and $T_{p}=\bigcap_{i=0}^{p-1} g^{i} T$. Then $\tilde{T}=T_{1} \supset T_{2} \supset \cdots \supset T_{p}$ gives a stratification. Note that $\operatorname{dim} \tilde{T}=\operatorname{dim} T_{1}>\operatorname{dim} T_{2}$. Note also that $T_{p}$ is $G$-invariant and contains all fixed points. By perturbing $\left.\bar{f}_{C}\right|_{\left.\bar{V}\right|_{T_{p}}} G$-equivariantly in the way similar to $\S 3,\left.\bar{f}_{C}\right|_{\left.\bar{V}\right|_{T_{p}}}$ comes to have no zero. (This is due to a dimensional reason.) Next perturb $\bar{f}_{C}$ on $\left.\bar{V}\right|_{T_{p-1} \backslash T_{p}} G$-equivariantly so that $\left.\bar{f}_{C}\right|_{\left.\bar{V}\right|_{T_{p-1} \backslash T_{p}}}$ has no zero. Successively perturb $\bar{f}_{C}$ on $\left.\bar{V}\right|_{T_{k} \backslash T_{k+1}}$ for $k>1 G$-equivariantly so that $\left.\bar{f}_{C}\right|_{\left.\bar{V}\right|_{T_{k} \backslash T_{k+1}}}$ has no zero. Finally, carry out a $G$-equivariant perturbation of $\left.\left.\bar{f}_{C}\right|_{\bar{V}}\right|_{\tilde{T}}$ outside $V_{T_{2}}$ to achieve the transversality with the zero-section. Since all zeros are on $\left.\bar{V}\right|_{\tilde{T} \backslash T_{2}}$, and $G$ acts freely on the set of zeros, the conclusion holds.

5. Examples. The purpose of this section is to give several examples. In order to apply Theorem 1.2 and Theorem 1.8 to concrete examples, we need to calculate coefficients $k_{j}^{l}$. Therefore we first discuss how to calculate coefficients $k_{j}^{l}$.

5.1. How to calculate $k_{j}^{l}$. Recall that we decomposed the fixed point set $J^{G}$ of the Jacobian torus into connected components: $J^{G}=J_{0} \cup \cdots \cup J_{K}$, and chose a point $t_{l}$ in each $J_{l}$. Fix a generator $g \in G$, and write $\hat{g}$ for the action of $g$ on the Spin ${ }^{c}$-structure $c$. For the origin $t_{0}=\left[A_{0}\right]$, by definition, it holds that $\hat{g} A_{0}=$ $A_{0}$. Therefore, we can calculate $k_{j}^{0}$ by the $G$-index formula such as $\operatorname{ind}_{g} D_{A_{0}}=$ 
(contributions from fixed points). First we briefly review the $G$-index formula. (See $[3,4,2,1]$.)

Let $X^{G}=X_{0} \cup X_{1} \cup \cdots \cup X_{N}$ be the decomposition of the fixed point set $X^{G}$ into connected components, where $X_{0}$ is assumed to be the component of the base point $x_{0}$. Then, the $G$-index formula for $t_{0}=\left[A_{0}\right] \in J^{G}$ is written as

$$
\operatorname{ind}_{g} D_{A_{0}}=\sum_{j=0}^{p-1} \zeta^{j} k_{j}^{0}=\sum_{n=0}^{N} \mathcal{F}_{n}^{0}(g),
$$

where $\zeta=\exp (2 \pi \sqrt{-1} / p)$ and each $\mathcal{F}_{n}^{0}(g)$ is a complex number associated to the component $X_{n}$ which is given as follows.

Let $L_{n}$ be the restriction of the determinant line bundle $L$ to $X_{n}$. Then $g$ acts on each fiber of $L_{n}$ as the multiplication with a complex number $\nu_{n}$ of absolute value 1. (In our case, $\nu_{n}$ is a $p$-th root of 1 .)

There are two cases with respect to the dimension of $X_{n}$. Since we assume the $G$-action is orientation-preserving, the dimensions of $X_{n}$ are even.

If $X_{n}$ is just a point $x_{n}$, the tangent space over $x_{n}$ is written as

$$
T_{x_{n}} X=N\left(\omega_{1}\right) \oplus N\left(\omega_{2}\right)
$$

where $N\left(\omega_{j}\right)$ is the complex 1-dimensional representation on which $g$ acts by multiplication with $\omega_{j}$. (In our case, $\omega_{j}$ is a $p$-th root of 1 .)

Then the number $\mathcal{F}_{n}^{0}(g)$ is given by,

$$
\mathcal{F}_{n}^{0}(g)=\nu_{n}^{\frac{1}{2}} \frac{1}{\omega_{1}^{1 / 2}-\omega_{1}^{-1 / 2}} \frac{1}{\omega_{2}^{1 / 2}-\omega_{2}^{-1 / 2}} .
$$

The right hand side is only defined up to sign. To determine the sign precisely, we need to see the $g$-action on the $\operatorname{Spin}^{c}$-structure $c$. When $G$ is the cyclic group of odd order $p$ and the $\operatorname{Spin}^{c}$-structure $c$ is $G$-equivariant, signs of $\omega_{i}^{1 / 2}$ and $\nu_{n}^{1 / 2}$ are determined by the rule that

$$
\left(\omega_{i}^{1 / 2}\right)^{p}=\left(\nu_{n}^{1 / 2}\right)^{p}=1
$$

(See $[2, \mathrm{p} .20]$.$) On the other hand, when p=2$, it is somewhat subtle problem to determine the sign precisely. (See [1].)

If $X_{n}$ is a 2-dimensional surface $\Sigma_{n}$, the restriction of the tangent bundle of $X$ to $\Sigma_{n}$ is written as

$$
\left.T X\right|_{\Sigma_{n}}=T \Sigma_{n} \oplus N(\omega)
$$

where $N(\omega)$ is the normal bundle of $\Sigma_{n}$ in $X$, and $g$ acts on the fiber of $N(\omega)$ as multiplication with $\omega$.

In this case, $\mathcal{F}_{n}^{0}(g)$ is given as,

$$
\mathcal{F}_{n}^{0}(g)=-\nu_{n}^{\frac{1}{2}} \cdot \frac{1}{2} \frac{\omega^{1 / 2}+\omega^{-1 / 2}}{\left(\omega^{1 / 2}-\omega^{-1 / 2}\right)^{2}}\left[\Sigma_{n}\right]^{2},
$$

where $\left[\Sigma_{n}\right]^{2}$ denotes the self intersection number of $\Sigma_{n}$. When $p$ is odd, (5.3) is valid with the sign if square roots are given by the rule (5.2).

In order to calculate $k_{j}^{l}$ for other $l$, we note the following lemma. 
Lemma 5.4. Let $g \in G$, and the action of $g$ on the $\operatorname{Spin}^{c}$-structure $c$ be denoted by $\hat{g}$. For a connection $A$ on $L$, if there exists $u \in \mathcal{G}_{0}$ which satisfies $\hat{g} A=u A$, i.e., $[A] \in J^{G}$, then we can define another action $\hat{g}^{\prime}$ of $g$ on $c$ so that $\hat{g}^{\prime} A=A$.

Proof. Consider the action $\left(u^{-1} \circ \hat{g}\right)$. Then $\left(u^{-1} \circ \hat{g}\right) A=A$. In particular, we have $\left(u^{-1} \circ \hat{g}\right)^{p} A=A$. Note that $\left(u^{-1} \circ \hat{g}\right)^{p}$ is an element of $\mathcal{G}_{0}$. Therefore $\left(u^{-1} \circ \hat{g}\right)^{p}=1$ Thus $\hat{g}^{\prime}:=(u)^{-1} \circ \hat{g}$ is a required action.

Thus, for any $t_{l}=\left[A_{l}\right] \in J^{G}$, we can redefine the $G$-action on $c$ so that $A_{l}$ is $G$-invariant. Hence, $k_{j}^{l}$ are also calculated by the $G$-index formula. However, the contributions from fixed points for the redefined action are different from the original ones as

$$
\operatorname{ind}_{g} D_{A_{l}}=\sum_{j=0}^{p-1} \zeta^{j} k_{j}^{l}=\sum_{n} \mathcal{F}_{n}^{l}(g),
$$

where $\mathcal{F}_{n}^{l}(g)$ are calculated as in (5.1) and (5.3) for the redefined $g$ action on $c$.

For different $l_{0}$ and $l_{1}$, the difference between $\mathcal{F}_{n}^{l_{0}}(g)$ and $\mathcal{F}_{n}^{l_{1}}(g)$ is given as follows. We can consider that a representation of $t_{l} \in J^{G}$ is given as a triplet $\left(S_{l}^{+}, \phi_{l}, A_{l}\right)$ of a $G$-spinor bundle $S_{l}^{+}$, a trivialization $\phi_{l}$ at $x_{0}$, and a $G$-invariant connection $A_{l}$ on the determinant line bundle $L_{l}=\operatorname{det} S_{l}^{+}$. For $l_{0}$ and $l_{1}$, the difference between $\left(S_{l_{0}}^{+}, \phi_{l_{0}}, A_{l_{0}}\right)$ and $\left(S_{l_{1}}^{+}, \phi_{l_{1}}, A_{l_{1}}\right)$ is given as a flat $G$-line bundle $\mathcal{L}_{l_{1} l_{0}}$ :

$$
\left(S_{l_{1}}^{+}, \phi_{l_{1}}, A_{l_{1}}\right)=\mathcal{L}_{l_{1} l_{0}} \otimes\left(S_{l_{0}}^{+}, \phi_{l_{0}}, A_{l_{0}}\right) .
$$

For each component $X_{n} \subset X^{G}$, the weight of $g$-action on the fiber of $\mathcal{L}_{l_{1} l_{0}}$ at $x_{n} \in X_{n}$ is given as a complex number $\lambda_{n}^{l_{1} l_{0}}$, which is a $p$-th root of 1 . Then the relation between $\mathcal{F}_{n}^{l_{0}}(g)$ and $\mathcal{F}_{n}^{l_{1}}(g)$ is given as

$$
\mathcal{F}_{n}^{l_{1}}(g)=\lambda_{n}^{l_{1} l_{0}} \mathcal{F}_{n}^{l_{0}}(g) .
$$

Before ending this subsection, we give a useful lemma for lifts of the $G$-action to a Spin ${ }^{c}$-structure.

Lemma 5.7. Let $G=\mathbb{Z}_{p}$, and $X$ be a closed oriented $G$-manifold which has no 2-torsion in $H_{1}(X ; \mathbb{Z})$. If the determinant line bundle of a $\operatorname{Spin}^{c}$-structure $c$ on $X$ is $G$-equivariant, then the $G$-action lifts to $c$, that is, $c$ is $G$-equivariant or $G$-action is of even or odd type with respect to $c$ when $p=2$.

Proof. If there is no 2 -torsion in $H_{1}(X ; \mathbb{Z})$, then there is a bijective correspondence between the set of equivalence classes of $\operatorname{Spin}^{c}$-structures and the set of equivalence classes of determinant line bundles. For $g \in G$, let $\bar{g}$ be the action of $g$ on $P_{\mathrm{SO}} \times{ }_{X} P_{\mathrm{U}(1)}$. Consider the 2-fold covering $P_{\mathrm{Spin}^{c}} \rightarrow P_{\mathrm{SO}} \times_{X} P_{\mathrm{U}(1)}$. Since $\bar{g}^{*} P_{\mathrm{Spin}^{c}}$ is isomorphic to $P_{\mathrm{Spin}^{c}}$, we can lift $\bar{g}$ to $P_{\mathrm{Spin}^{c}}$. Therefore the $G$-action on $P_{\mathrm{SO}} \times_{X} P_{\mathrm{U}(1)}$ lifts to a $\hat{G}$-action on $P_{\text {Spin }^{c}}$.

5.2. An example of application in the case when $G=\mathbb{Z}_{2}$. The next proposition which is an application of Theorem 1.8 is also a generalization of Fang's result. (Compare with Corollary 4 of [10].) However, this is not a "new result", for this can be proved by the adjunction inequality. (See Example 5.9.) Nevertheless, we state this as an example of application.

Proposition 5.8. Let $G=\mathbb{Z}_{2}$, and $X$ be a closed oriented 4-dimensional $G$ manifold with $b_{+} \geq 2$ and $b_{+}^{G} \geq 1$, and suppose that $H_{1}(X ; \mathbb{Z})$ has no 2-torsion. 
Suppose that there is a $\mathrm{Spin}^{c}$-structure $c$ whose determinant line bundle is trivial, and $\mathrm{SW}_{X}(c) \not \equiv 0 \bmod 2$. Let $d(c)$ be as in (2.4). If the G-action has no isolated fixed point, then the following inequality holds:

$$
\begin{aligned}
& 1-b_{1}+b_{+} \geq 2\left(1-b_{1}^{G}+b_{+}^{G}\right), \text { when } d(c) \equiv 0 \bmod 4, \\
& 1-b_{1}+b_{+} \geq 2\left(-b_{1}^{G}+b_{+}^{G}\right), \text { when } d(c) \equiv 2 \bmod 4 .
\end{aligned}
$$

Proof. Note that $c$ is the $\operatorname{Spin}^{c}$-structure which is determined by a Spin-structure. Since the determinant line bundle $L$ is trivial, we can define a $G$-action on $L$ which is the product of the $G$-action on $X$ and trivial action on fiber. Therefore the $G$-action lifts to $c$ by Lemma 5.7. The lifted action may be of odd or even type with respect to $c$. We take the trivial flat connection $A_{0}$ on $L$ as the origin of the Jacobian torus $J$. As is known widely, a $G$-action is of even type if and only if the fixed point set is isolated. On the other hand, a $G$-action is of odd type if and only if the fixed point set is 2-dimensional. (See e.g. [1].) Therefore, if the $G$-action is of even type, then it must be free by the assumption.

Suppose that the $G$-action is of odd type. By the $G$-index formula (put $\omega=-1$ and $\nu_{n}=1 \mathrm{in}(5.3)$ ), we have $\mathcal{F}_{n}^{0}(g)=0$ for any component $X_{n}$ of $X^{G}$. The relation (5.6) implies $\mathcal{F}_{n}^{l}(g)=0$ for any $l$ and $n$.

Therefore, we have $k_{1}^{l}=k_{3}^{l}=\frac{1}{2}$ ind $D_{A_{0}}$ for any $l$. By Theorem 1.8 with the assumption of mod 2 non-vanishing of $\mathrm{SW}_{X}(c)$, it holds that, for any partition $\left(d_{1}, d_{3}\right)$ of $d(c) / 2$, there exist $l$ and $j$ which satisfy

$$
2 k_{j}^{l} \geq 2 d_{j}+1-b_{1}^{G}+b_{+}^{G} .
$$

Therefore we have

$$
\begin{aligned}
& \text { ind } D_{A_{0}} \geq \frac{d(c)}{2}+1-b_{1}^{G}+b_{+}^{G}, \quad \text { when } d(c) \equiv 0 \bmod 4, \\
& \text { ind } D_{A_{0}} \geq\left(\frac{d(c)}{2}-1\right)+1-b_{1}^{G}+b_{+}^{G}, \text { when } d(c) \equiv 2 \bmod 4 .
\end{aligned}
$$

On the other hand, from the formula of the dimension of the moduli (2.4), we have

$$
\text { ind } D_{A_{0}}=\frac{1}{2}\left(d(c)+1-b_{1}+b_{+}\right) \text {. }
$$

This formula with above two inequality implies the proposition.

In the even case, the $G$-action should be free. In the free case, the theorem is obvious from the Lefschetz formula and the $G$-signature formula.

ExAmple 5.9. Concrete examples of $G=\mathbb{Z}_{2}$-actions are given as follows. Let $X$ be the $K 3$ surface of Fermat type, $X=\left\{\left[z_{0}, z_{1}, z_{2}, z_{3}\right] \in \mathbb{C P}^{3} \mid z_{0}^{4}+z_{1}^{4}+z_{2}^{4}+z_{3}^{4}=0\right\}$. Let $G$ act on $X$ by the permutation of two coordinates. Then the fixed point set is a complex curve $C$ whose genus is 3 and self-intersection number is 4 .

Another example of $b_{1}>0$ is 4 -torus. Let $X$ be the direct product of two copies of 2 -torus. Let $G$ act on the first 2 -torus by multiplication by -1 , and on the second trivially. The fixed point set consists of four 2-tori whose self-intersection number are 0 .

Let us verify Proposition 5.8 for these examples. It is well-known that $\mathrm{SW}_{X}\left(c_{0}\right)=$ \pm 1 for the $K 3$ surface and the 4 -torus [19]. Note that, for a (V-)manifold $Y$, it holds that

$$
1-b_{1}(Y)+b_{+}(Y)=\frac{1}{2}(\chi(Y)+\operatorname{Sign}(Y))
$$


Therefore, by using the Lefschetz formula and the $G$-signature theorem, we have

$$
\begin{aligned}
1-b_{1}^{G}+b_{+}^{G} & =\frac{1}{2}(\chi(X / G)+\operatorname{Sign}(X / G)) \\
& =\frac{1}{2}\left\{\frac{1}{2}(\chi(X)+\chi(C))+\frac{1}{2}\left(\operatorname{Sign}(X)+[C]^{2}\right)\right\} \\
& =\frac{1}{2}\left\{\frac{1}{2}(\chi(X)+\operatorname{Sign}(X))\right\} \\
& =\frac{1}{2}\left(1-b_{1}+b_{+}\right) .
\end{aligned}
$$

We use the adjunction formula at the third equality. From this calculation, we see that the adjunction inequality $\chi(C)+[C]^{2} \leq 0$ proves Proposition 5.8.

REMARK 5.10. We can construct similar $G$-actions on homology 4-tori obtained by the 'knot surgery' construction according to [17] and [11]. (See also Example 5.11.)

5.3. Examples of the case when $G=\mathbb{Z}_{3}$. This subsection treats with the case when $G=\mathbb{Z}_{3}$. In the following, we assume that the $G$-action is pseudofree, that is, the $G$-action has only isolated fixed points. In such a case, fixed points are classified into two types of representations:

- The type $(+):(1,2)=(2,1)$.

- The type $(-):(1,1)=(2,2)$.

Let $m_{+}$be the number of fixed points of the type $(+)$, and $m_{-}$be that of the type $(-)$.

We give examples of pseudofree $G$-actions which imply the mod-3 vanishing of Seiberg-Witten invariants.

EXAMPLE 5.11. Let $X$ be the direct product of a 2-torus and a Riemann surface of genus $3 h(h \geq 1)$. We construct a $G$-action on $X$ as follows. Let us consider the lattice $\mathbb{Z} \oplus \zeta \mathbb{Z} \subset \mathbb{C}$, where $\zeta=\exp (2 \pi \sqrt{-1} / 3)$, and let $T_{1}$ be the 2-torus $\mathbb{C}_{1} /(\mathbb{Z} \oplus \zeta \mathbb{Z})$ with a $G$-action, where the $G$-action is given by the multiplication by $\zeta$. Next consider a 2 -sphere, and let $G$ act on the 2 -sphere by $2 \pi / 3$-rotation. Taking a free point $q$ on the 2-sphere, and glueing 3 copies of a Riemann surface of genus $h$ to the 2 -sphere at three points $q, g q, g^{2} q$, we obtain a Riemann surface $\Sigma_{3 h}$ of genus $3 h$ with a $G$-action. Let $X$ be $T_{1} \times \Sigma_{3 h}$ with the diagonal $G$-action.

Now let us examine Theorem 1.2. First note that the fixed point set of $T_{1}$ consists of three points $p_{0}, p_{1}$ and $p_{2}$, and all of them have same type of representation: $T\left(T_{1}\right)_{p_{n}} \cong \mathbb{C}_{1}$. On the other hand, $\Sigma_{3 h}$ have two fixed points $q_{+}$and $q_{-}$, and they have opposite representations each other. (We assume that $q_{+}$is the fixed point such that $T\left(\Sigma_{3 h}\right)_{q_{+}} \cong \mathbb{C}_{2}$. ) Therefore, $X$ has six fixed points, and three of them are of the type $(+)$, and the other three are of the type $(-)$.

Note that $\chi(X)=\operatorname{Sign}(X)=0$ and $X$ is spin. We take the $\operatorname{Spin}^{c}$-structure $c_{0}$ which is determined by a Spin-structure. Note that $d\left(c_{0}\right)=0$. We consider the $G$-action on $c_{0}$ which induces the $G$-action on the determinant line bundle $L$ which is the product of the $G$-action on $X$ and the trivial action on fiber. Take the trivial flat connection $A_{0}$ on $L$ as the origin of the Jacobian torus $J_{X}$.

The Jacobian $J_{X}$ is of the form $J_{X}=J_{T_{1}} \times J_{\Sigma_{3 h}}$. For a fixed point $t=(a, b) \in J_{X}^{G}$, the corresponding flat $G$-bundle $\mathcal{L}_{t}$ is written as $\mathcal{L}_{t}=\pi_{1}^{*} \mathcal{L}_{a} \otimes \pi_{2}^{*} \mathcal{L}_{b}$, where $\pi_{1}$ (resp. $\pi_{2}$ ) is the projection to $T_{1}$ (resp. $\Sigma_{3 h}$ ), and $\mathcal{L}_{a}$ is the flat $G$-bundle on $T_{1}$ associated to $a \in J_{T_{1}}^{G}$ and $\mathcal{L}_{b}$ is similar. 
Now let us attempt to classify flat $G$-bundles on a Riemann surface. Temporarily, we consider more general situation that $G_{p}=\mathbb{Z}_{p}$ acts pseudofreely on a Riemann surface $\Sigma_{g}$ of genus $g$. Let $\left\{p_{n}\right\}$ be the fixed point set. Consider a divisor $D$ on $\Sigma_{g}: D=\sum_{n} d_{n} p_{n}$. Then we can construct a $G_{p}$-line bundle $\mathcal{L}_{D}$ on $\Sigma_{g}$ which satisfy $\left.\mathcal{L}_{D}\right|_{p_{n}} \cong\left(\left.T \Sigma_{g}\right|_{p_{n}}\right)^{\otimes d_{n}}$. Note that $c_{1}\left(\mathcal{L}_{D}\right)=\sum d_{n}$. In this situation, we can prove the following.

Proposition 5.12. Let $\mathcal{L}$ be a $G_{p}$-line bundle on $\Sigma$ which satisfy $\left.\mathcal{L}\right|_{p_{n}} \cong$ $\left(\left.T \Sigma_{g}\right|_{p_{n}}\right)^{\otimes d_{n}}$. Then $c_{1}(\mathcal{L}) \equiv c_{1}\left(\mathcal{L}_{D}\right) \bmod p$.

Proof. Let us consider the line bundle $\mathcal{L} \otimes \mathcal{L}_{D}^{-1}$. Then there is a line bundle $\overline{\mathcal{L}}$ on $\Sigma_{g} / G_{p}$ which satisfies $\pi^{*} \overline{\mathcal{L}} \cong \mathcal{L} \otimes \mathcal{L}_{D}^{-1}$, where $\pi: \Sigma_{g} \rightarrow \Sigma_{g} / G_{p}$ is the quotient map. Noting that $c_{1}\left(\mathcal{L} \otimes \mathcal{L}_{D}^{-1}\right)=\pi^{*} c_{1}(\mathcal{L})$, and $\pi^{*}: H^{2}\left(\Sigma_{g} / G_{p} ; \mathbb{Z}\right) \rightarrow H^{2}\left(\Sigma_{g} ; \mathbb{Z}\right)$ is multiplication by $p$, we have the proposition.

Let us apply Proposition 5.12 to $\Sigma_{3 h}$ with the $G$-action. Since the fixed point set is $\left\{q_{+}, q_{-}\right\}$, the divisor $D$ is of the form $D=d_{+} q_{+}+d_{-} q_{-}$. Since $\mathcal{L}_{b}$ is trivial, we have $0=c_{1}\left(\mathcal{L}_{b}\right) \equiv d_{+}+d_{-} \bmod 3$. Therefore, the following holds.

Lemma 5.13. For any $b \in J_{\Sigma_{3 h}}^{G}, \mathcal{L}_{b}$ is isomorphic to $\mathcal{L}_{D}$ such that $D=0$ or $q_{+}-q_{-}$or $2 q_{+}-2 q_{-}$.

For $b \in J_{\Sigma_{3 h}}^{G}$, let us denote the weight of the $G$-action on the fiber of $\mathcal{L}_{b}$ at $q_{+}$ (resp. $q_{-}$) by $\lambda_{+}^{b}$ (resp. $\lambda_{-}^{b}$ ). Similarly, for $a \in J_{T_{1}}^{G}$, denote the weight of $\mathcal{L}_{a}$ at $p_{i} \in T_{1}^{G}$ by $\lambda_{i}^{a}$. Note that $\mathcal{F}_{\left(p_{i}, q_{ \pm}\right)}^{(0,0)}(g)$ for the origin $(0,0) \in J_{X}^{G}$ at $\left(p_{i}, q_{ \pm}\right) \in X^{G}$ is given by $\mathcal{F}_{\left(p_{i}, q_{ \pm}\right)}^{(0,0)}(g)= \pm \frac{1}{3}$. (See (5.1).) Therefore $\mathcal{F}_{\left(p_{i}, q_{ \pm}\right)}^{(a, b)}(g)$ for $(a, b) \in J_{X}^{G}$ at $\left(p_{i}, q_{ \pm}\right)$is written as

$$
\mathcal{F}_{\left(p_{i}, q_{ \pm}\right)}^{(a, b)}(g)= \pm \frac{1}{3} \lambda_{i}^{a} \lambda_{ \pm}^{b} .
$$

By Lemma 5.13, we have $\lambda_{+}^{b}=\lambda_{-}^{b}$.Hence we obtain

$$
\sum_{x \in X^{G}} \mathcal{F}_{x}^{(a, b)}(g)=\frac{1}{3}\left(\sum_{i=0}^{2} \lambda_{i}^{a}\right)\left(\lambda_{+}^{b}-\lambda_{-}^{b}\right)=0 .
$$

Similarly we obtain

$$
\sum_{x \in X^{G}} \mathcal{F}_{x}^{(a, b)}\left(g^{2}\right)=0
$$

for any $(a, b) \in J_{X}^{G}$.

By (5.14) and (5.15), the $G$-index formula for the Dirac operator of $t_{l}=\left[A_{l}\right] \in J^{G}$ is given as

$$
\begin{aligned}
\operatorname{ind}_{g} D_{A_{l}} & =k_{0}^{l}+\zeta k_{1}^{l}+\zeta^{2} k_{2}^{l}=0, \\
\operatorname{ind}_{g^{2}} D_{A_{l}} & =k_{0}^{l}+\zeta^{2} k_{1}^{l}+\zeta k_{2}^{l}=0, \\
\operatorname{ind}_{1} D_{A_{l}} & =k_{0}^{l}+k_{1}^{l}+k_{2}^{l}=-\frac{1}{8} \operatorname{Sign}(X)=0 .
\end{aligned}
$$

Solving these equations, we obtain

$$
k_{0}^{l}=k_{1}^{l}=k_{2}^{l}=0 .
$$


Now let us check that inequalities (1.3) are satisfied. First let us compute $1-$ $b_{1}^{G}+b_{+}^{G}$. The Lefschetz formula implies that

$$
\chi(X / G)=\frac{1}{3}\left(\chi(X)+2\left(m_{+}+m_{-}\right)\right) .
$$

On the other hand, the $G$-signature theorem (Cf.[1]) implies that

$$
\begin{gathered}
\operatorname{Sign}(g, X)=\operatorname{Sign}\left(g^{2}, X\right)=\frac{1}{3}\left(m_{+}-m_{-}\right), \\
\operatorname{Sign}(X / G)=\frac{1}{3}\left\{\operatorname{Sign}(X)+\frac{2}{3}\left(m_{+}-m_{-}\right)\right\} .
\end{gathered}
$$

Since $\chi(X)=\operatorname{Sign}(X)=0$, we have,

$$
1-b_{1}^{G}+b_{+}^{G}=\frac{1}{2}(\chi(X / G)+\operatorname{Sign}(X / G))=\frac{1}{9}\left(4 m_{+}+2 m_{-}\right)=2 .
$$

Since the dimension of the moduli $d\left(c_{0}\right)$ is 0 , all $d_{j}$ in (1.3) should be 0 . Therefore inequalities (1.3) are satisfied as,

$$
2 k_{j}^{l}=0<2=1-b_{1}^{G}+b_{+}^{G},
$$

for any $j, l$, and hence Theorem 1.2 implies that $\mathrm{SW}_{X}\left(c_{0}\right) \equiv 0 \bmod 3$.

On the other hand, we can calculate the Seiberg-Witten invariants of $X_{g}=T^{2} \times$ $\Sigma_{g}$. The answer is given as follows: for the $\operatorname{Spin}^{c}$-structure $c_{0}$ which is determined by a Spin-structure,

$$
\mathrm{SW}_{X_{g}}\left(c_{0}\right)= \pm\left(\begin{array}{c}
2 g-2 \\
g-1
\end{array}\right) .
$$

It is easy to see that this is divisible by 3 if $g=3 h$. Thus, Theorem 1.2 holds.

There are several methods to prove (5.20). One method is Witten's calculation [22, pp.786-792]. The canonical divisor of $X_{g}$ is written as $c_{1}(K)=(2 g-2) P . D .[T \times$ $p t]$. For a generic choice of $\eta \in H^{0}\left(X_{g}, K\right)$, a Seiberg-Witten solution corresponds to a factorization $\eta=\alpha \beta$, where $\alpha$ and $\beta$ are holomorphic sections of $K^{1 / 2} \otimes L^{ \pm 1}$. Since $L$ of our case is trivial, the number of possibilities of factorizations $\eta=\alpha \beta$ coincides with the right hand side of (5.20). Furthermore, we can see that all solutions have same sign also by [22].

An alternative way to prove (5.20) is as follows. First consider $X_{g}$ as $S^{1} \times M$, where $M=S^{1} \times \Sigma_{g}$. Next determine the Seiberg-Witten invariants of $M$ by, for instance, Turaev torsion of $M$. Then use the formula $\mathrm{SW}_{S^{1} \times M}(\tilde{c})=\mathrm{SW}_{M}(c)$ where $\tilde{c}$ is the pull-back of $c$. When $g \geq 2$, Turaev torsion of $S^{1} \times \Sigma_{g}$ is written as $\pm(t-1)^{2 g-2}$, where $t$ is the homology class represented by $S^{1}$, and $c_{0}$ corresponds to the term of order $g-1$. (See [20, pp.93-96].)

REMARK 5.21. Similar examples can be constructed via the 'knot surgery' construction of Fintushel and Stern [11]. Remove three copies of $T^{2} \times D^{2}$ from $X=T^{2} \times \Sigma_{3 h}$ which are mapped to each other by the $G$-action, and denote the resulting manifold by $X^{\prime}$. According to [11], let $K$ be a knot in $S^{3}$, and $E_{K}$ be the exterior. Then glueing $S^{1} \times E_{K}$ to each boundary of $X^{\prime}$ gives an example. This manipulation changes the Seiberg-Witten invariant by a multiple of 3 [11]. 
REMARK 5.22. We can construct an example of $G$-action such that the SeibergWitten invariant does not vanish modulo 3 and there exists $l$ for which (1.3) does not hold. Let $T_{i}$ be the 2 -torus $\mathbb{C}_{i} /(\mathbb{Z} \oplus \zeta \mathbb{Z})$ with the $G$-action given by the multiplication by $\zeta^{i}(i=1,2)$. Remove a small $G$-invariant neighborhood of a fixed point of each $T_{i}$. Since fixed points of $T_{1}$ and $T_{2}$ have opposite representations, we can glue their boundaries $G$-equivariantly, and the resulting manifold is a Riemann surface $\Sigma_{2}$ of genus 2 with a $G$-action whose fixed point set consists of four points. Now consider the 4-manifold $T_{1} \times \Sigma_{2}$ with the diagonal $G$-action. Then we can prove that this is a required example.

\section{REFERENCES}

[1] M. F. Atiyah And R. Bott, A Lefschetz fixed point formula for elliptic complexes II Applications, Ann. Math., 88 (1968), pp. 451-491.

[2] M. F. AtiYah And F. Hirzebruch, Spin-manifolds and group actions, Essays on topology and related topics, Memoires dédié à George de Rham (ed. A. Haefliger and R. Narashimhan), Springer-Verlag (1970), pp. 18-28.

[3] M. F. Atiyah and G. B. Segal, The index of elliptic operators: II, Ann. Math., 87 (1968), pp. 531-545.

[4] M. F. Atiyah And I. M. Singer, The index of elliptic operators: III, Ann. Math., 87 (1968), pp. 546-604.

[5] S. BALDRIDGe, Seiberg-Witten vanishing theorem for $S^{1}$-manifolds with fixed points, Pacific J. Math., 217 (2004), pp. 1-10.

[6] S. Bauer and M. Furuta, A stable cohomotopy refinement of Seiberg-Witten invariants: I, Invent. Math., 155 (2004), pp. 1-19.

[7] J. Bryan, Seiberg-Witten theory and $\mathbb{Z} / 2^{p}$ actions on spin 4 -manifolds, Math. Res. Lett., 5 (1998), pp. 165-183.

[8] Y. S. Cho And Y. H. Hong, Seiberg-Witten invariants and (anti-)symplectic involutions, Glasgow Math. J., 45 (2003), pp. 401-413.

[9] F. FANG, Smooth group actions on 4-manifolds and the Seiberg-Witten theory, Diff. Geom. Appl., 14 (2001), pp. 1-14.

[10] F. FAnG, Smooth group actions on 4-manifolds and Seiberg-Witten invariants, International J. Math., 9:8 (1998), pp. 957-973.

[11] R. Fintushel And R. Stern, Knots, links, and 4-manifolds, Invent. Math., 134 (1998), pp. 363400.

[12] M. Furuta, Monopole equation and the $\frac{11}{8}$-conjecture, Math. Res. Lett., 8 (2001), pp. 279-291.

[13] M. Furuta, Stable homotopy version of Seiberg-Witten invariant, preprint, the Max-PlanckInstitut für Mathematik (http://www.mpim-bonn.mpg.de/), MPIM1997-110.

[14] X. Liu and N. Nakamura, Pseudofree $\mathbb{Z} / 3$-actions on K3 surfaces, preprint.

[15] N. Nakamura, A free $\mathbb{Z}_{p}$-action and the Seiberg-Witten invariants, J. Korean Math. Soc., 39:1 (2002), pp. 103-117.

[16] Y. RuAn And S. WAng, Seiberg-Witten invariants and double covers of 4-manifolds, Comm. Anal. Geom., 8:3 (2000), pp. 477-515.

[17] D. Ruberman and S. Strle, Mod 2 Seiberg-Witten invariants of homology tori, Math. Res. Lett., 7 (2000), pp. 789-799.

[18] M. SZYMiK, Bauer-Furuta invariants and Galois symmetries, preprint.

[19] C. Taubes, The Seiberg-Witten invariants and symplectic forms, Math. Res. Lett., 1 (1994), pp. $769-796$.

[20] V. Turaev, Torsions of 3-dimensional manifolds, Progress in Mathematics, vol. 208, Birkhäuser.

[21] S. WANG, A vanishing theorem for Seiberg-Witten invariants, Math. Res. Lett., 2 (1995), pp. 305-310.

[22] E. Witten, Monopoles and four-manifolds, Math. Res. Lett., 1 (1994), pp. 769-796. 